\title{
Catalogue of Chrysomelini Species (Coleoptera: Chrysomelidae: Chrysomelinae) from the New Leaf Beetles Collection of "Grigore Antipa" National Museum of Natural History (Bucharest) (Part III)
}

\author{
Sanda MAICAN ${ }^{1, *}$, Rodica SERAFIM ${ }^{2}$ \\ ${ }^{1}$ Institute of Biology Bucharest of Romanian Academy, 296 Splaiul Independenţei, 060031 Bucharest, \\ P.O. Box 56-53, Romania. \\ 2"Grigore Antipa" National Museum of Natural History, 1 Kiseleff, 011341 Bucharest, Romania. \\ "corresponding author, e-mail: sanda.maican@ibiol.ro
}

Received: April 15, 2018; Accepted: June 12, 2018; Available online: June 25, 2018; Printed: June 30, 2018

\begin{abstract}
The paper presents data on the Palaearctic species of Chrysomelini tribe (Coleoptera: Chrysomelidae: Chrysomelinae) preserved in the new leaf beetles Collection of "Grigore Antipa" National Museum of Natural History (Bucharest). A total of 4,442 specimens, belonging to 21 species from eight genera of tribe Chrysomelini, are listed. Among the rare species in Romanian fauna, stored in this collection, Chrysomela lapponica Linnaeus, 1758, Prasocuris junci (Brahm, 1790) and Chrysomela cuprea Fabricius, 1775 should be mentioned.
\end{abstract}

Key words: Chrysomelidae, Chrysomelinae, Chrysomelini tribe, collection, "Grigore Antipa" National Museum of Natural History Bucharest.

\section{INTRODUCTION}

Subfamily Chrysomelinae (Coleoptera: Chrysomelidae) includes aproximately 130 genera with about 3000 species and subspecies. Most of them are widely distributed in dried tropical and temperate areas of the world, but several species occur in Arctic tundra and sandy desert. Chrysomelinae taxa are classified in two tribes, Chrysomelini and Timarchini. The monotypic tribe Timarchini occurs in the Western Palearctic and Nearctic, whereas the Chrysomelini is widespread (Bouchard et al., 2011; Reid, 2014). According to the Catalogue of Palaearctic Coleoptera (Kippenberg, 2010), 23 species of eight genera of tribe Chrysomelini are reported in Romanian fauna.

The "Grigore Antipa" Museum included the Chrysomelini material in the Collection of Palaearctic Coleoptera (some of the data have been published) and in the Collection of Chrysomelidae, which was recently formed (since 2016). In the first two parts of the Catalogue of New Chrysomelidae Collection, data on 64 species of the Donaciinae (Donaciini, Haemoniini and Plateumarini tribes), Criocerinae, and Cassidinae (Cassidini and Hispini tribes) were published (Maican \& Serafim, 2016, 2017). 


\section{MATERIAL AND METHODS}

The new Chrysomelini collection, preserved in the new Chrysomelidae collection of the "Grigore Antipa" National Museum of Natural History, Bucharest, comprises:

- material that was not included in the Collection of Palaearctic Coleoptera, containing specimens from Deszö (Dénes) Kenderessy (1846-1881?), Edmund Reitter (1845-1920), Arnold Lucien Montandon (1852-1922), Friedrich Deubel (1845-1933), Richard Canisius (1872-1934), and Emil Váradi collections. The material from "Deszö Kenderessy" collection, an amateur entomologist, was collected at the end of the $19^{\text {th }}$ century from Transylvania (Haţeg Depression, Hunedoara County). The "Arnold Lucien Montandon" collection consists in material collected at the end of the $19^{\text {th }}$ century and the beginning of the $20^{\text {th }}$ one from Romania: the royal estate of Broşteni, the Carpathian Mountains (Suceava County), Bârlad County, Comana Forest (Giurgiu County), Dobrogea region and France. A part of the material preserved in the "Fridrich Deubel" collection originates especially in the surroundings of Braşov. The specimens from the "Emil Váradi" collection were collected from Maramureș and Banat regions, and Harghita County in the first half of the $20^{\text {th }}$ century. The old collection includes also specimens collected by Richard Canisius (painter of the "Grigore Antipa" Museum during 1907-1934).

- material collected by the team of specialists of the "Grigore Antipa" Museum and their collaborators during different research projects on the Romanian fauna (1951-2017);

- material from Dr. Nicolae Săvulescu collection which was included in the Museum patrimony in 1992, after his death;

- specimens donated by Igor Ceianu (1925-2000), Mihai Şerban Procheş, Viorel Ungureanu, Vladimir Brădescu, Victor Gheorghiu;

- material collected during the scientific expeditions organized by "Grigore Antipa" Museum in the surroundings of the Mediterranean basin: "Focida" 2006 (Turkey) and Sabka "2010" (Syria).

The paper presents the results of the study of the Chrysomelini material (subtribes Chrysomelina, Gastrophysina, Phratorina and Prasocurina) preserved in the new Chrysomelidae collection of the "Grigore Antipa" National Museum of Natural History, Bucharest.

A part of the material of Chrysomelidae was published by Negru \& Roşca (1967), Negru (1968), Hoinic (1994), Maican \& Serafim (2001, 2004, 2009, 2012), Maican (2006, 2007 a, b), Serafim \& Maican (2004, 2008, 2011), Ungureanu et al. (2008), Stan et al. (2016).

The species identification was made by Atena Roşca ${ }^{1}$, Igor Ceianu ${ }^{2}$, Cristina Hoinic $^{3}$, Sanda Maican, Rodica Serafim, and Ştefan Negru4.

The specimens were identified based on the keys of Warchałowski papers (2003, 2010).

\footnotetext{
'Atena Roşca (1930-1976), worked at the Museum during 1954 to 1976.

${ }^{2}$ Igor Ceianu (1925-2000), Romanian specialist in the field of plant protection.

${ }^{3}$ Cristina Hoinic (1967-1997), worked at the museum during 1992 to 1997.

${ }^{4}$ Ştefan Negru (1923-1970), worked at the Museum during 1963 to 1970.
} 
The stereomicroscope Zeiss Stemi 2000-C was used to identify the specimens, and photos were taken with a Canon EOS 5D Mark IV digital camera.

The systematics and nomenclature are in accordance with the Catalogue of the Palaearctic Coleoptera (Kippenberg, 2010).

For each recorded species, information on the collecting date and place (presented in chronological order), the number of the specimens, the name of the collectors and the host plants are provided.

Abbreviations:

coll. - collection; f.c. - forest chalet; Mt. (Mts) - Mountain/Mountains; m a.s.1. - meters above sea level; spec. (s) - specimen/specimens.

Names of the collectors:

A.V. - Adriana Văraru; A.L. - Alexandra Levardă; A.K. - Ana-Maria Krapal; A.P. - Angela Petrescu; A.L.M. - Arnold Lucien Montandon; A.R. - Atena Roşca; A.P.G. - Aurelian Popescu-Gorj; A.S. - Aurora Stănescu; C.P. - Corneliu Pârvu; C.A. - Costică Adam; C.B. - Cristina Ban (Calefariu); C.C. - Cristina Constantinescu; Ct.C. - Constantin Ciubuc; D.D. - Dan Dumitrescu; D.K. - Deszo (Denes) Kenderessy; D.L. - Dochița Lupu; D.R. - Dorel Ruști; D.S. - Dragoş Scăunaşu; D.M. - Dumitru Murariu; Du.S. - Dumitru Stan; Ed.R. - Edmund Reitter; E.I. - Elena Iorgu (Pisică); E.R. - Elena Rusu; E.V. - Emil Váradi; Fr.D. - Friedrich Deubel; G.A. - Gabriela Andrei; G.C. - Gabriel Chișamera; I.Ca. - Ioana Cacoveanu; I.C. - Ileana Cursaru; I.M. - Ioana Matache; I.C. - Igor Ceianu; I.S. - Igor Sienkiewicz; I.D. - Ion Drăghia; I.N. - Ioan Nemeş; I.L. - Iosif Lepși; I.Şt.I. - Ionuţ Ștefan Iorgu; Iu. M. - Iuliana Medve; L.M. - Liviu Moscaliuc; L.O.P. - Luis Ovidiu Popa; M.V. - Maria Vasiliu; M.T. Marilena Taloi; M.W. - Medeea Weinberg; M.S. - Melanya Stan; M.B. - Mihai Băcescu; M. S.P. - Mihai Şerban Procheș; Mi.S. - Mihai Stănescu; M.A. - Mircea Andrei; M.M. - M. Manoliu; M.St. - Mihail Stan; N.R. - Năstase Răduleț; N.G. - Nicolae Găldean; N.S. - Nicolae Săvulescu; R.C. - Richard Canisius; R.S. - Rodica Serafim; R.Z. - Răzvan Zaharia; S.G.- Sorin Grigore; S. - Spătaru; Șt.N. Ştefan Negru; V.A. - Vasile Andreescu; V.G. - Victor Gheorghiu; V.I.R. - Victoria Iuga Raica; V.B. - Vladimir Brădescu; Vi.G. - Viorel Gavril; V.U. - Viorel Ungureanu; X.S.P. - Xenia Scobiola Palade. Abbreviations of the county names in Romania (Fig. 1):

AB - Alba; AG - Argeș; BC - Bacău; BN - Bistrița Năsăud; BR - Brăila; BH - Bihor; BV Braşov; BZ - Buzău; CS - Caraş Severin; CL - Călăraşi; CJ - Cluj; CT - Constanţa; DB - Dâmbovița; DJ - Dolj; GL - Galați; GJ - Gorj; GR - Giurgiu; HD - Hunedoara; HR - Harghita; IF - Ilfov; IL - Ialomița; IS - Iaşi; MH - Mehedinți; MM - Maramureş; MS - Mureş; NT - Neamț; PH - Prahova; SB - Sibiu; SJ - Sălaj; SM - Satu Mare; SV - Suceava; TL - Tulcea; TR - Teleorman; VS - Vaslui; VL - Vâlcea; VN - Vrancea. București - Bucharest (capital city of Romania).

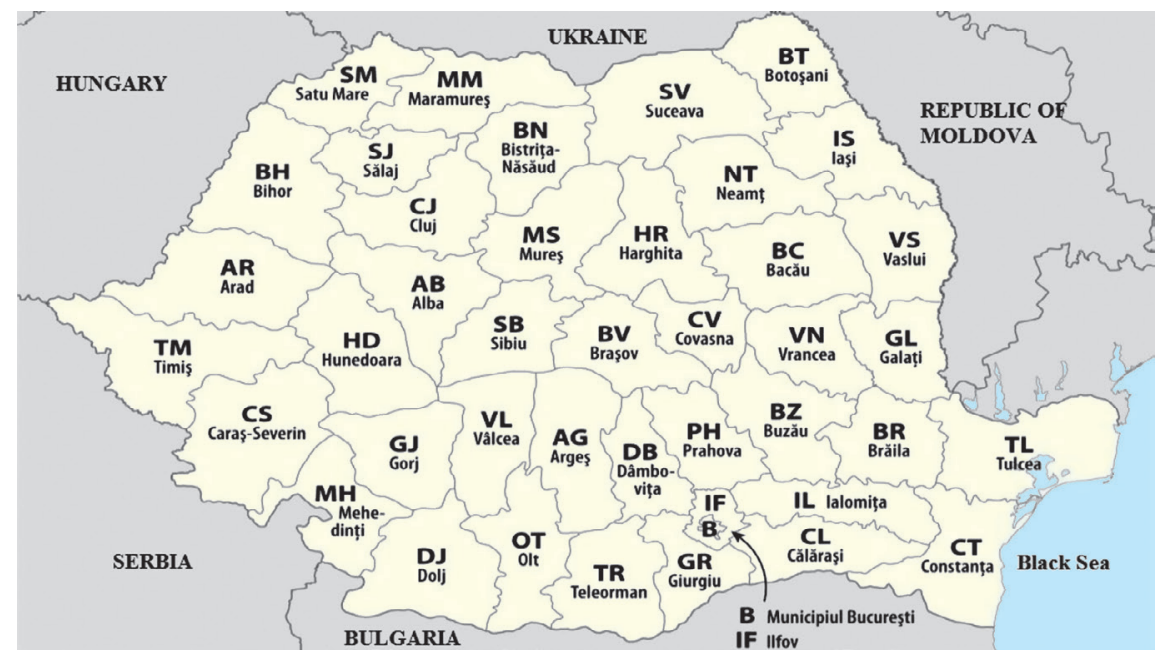

Fig. 1 - The map of the Romanian counties (modified from http://www.oradesibiu.ro) 


\section{RESULTS}

Based on the material examined so far, the new Chrysomelidae Collection of "Grigore Antipa" National Museum of Natural History from Bucharest includes 4,442 specimens, belonging to 21 species of eight genera of the Chrysomelini tribe, presented below.

Family Chrysomelidae Latreille, 1802

Subfamily Chrysomelinae Latreille, 1802

Tribe Chrysomelini Latreille, 1802

Subtribe Chrysomelina Latreille, 1802

Chrysomela Linnaeus, 1758

Chrysomela collaris Linnaeus, 1758

Material: 2 specs, Haţeg (Hatzeg) (HD), without other data, coll. D.K.; 1 spec., Transylvania, without other data, coll. D.K.; 5 specs, Mireşu Mare (Nagynyires) (MM), coll. E.V.; 2 specs, Buhuși (BC), 30.V.1944, coll. I.C.; Câmpulung Moldovenesc (SV), 30.V.1949, coll. I.C. (2 specs), 31.VII.1963, coll. I.C. (3 specs); 1 spec., Gurghiu (MS), 12.VII.1952, coll. I.C.; 1 spec., Şimleu Silvaniei (SJ), 1.VIII.1957, X.S.P.; 2 specs, Borsec (HR), 18.VI.1960, coll. I.C.; 2 specs, Adjud (VN), 22.III.-19.VI.1961; 6 specs, Frasin (SV), 24.-25.VII.1962, coll. I.C.; 2 specs, Eșelnița Valley (MH), 3.VI.1968, X.S.P.; 6 specs, Slătioara Secular Forest (SV), V.1983, M.B.; 3 specs, Cămărzana (SM), 19.V.1987, R.S.; 10 specs, Strâmtura (MM), 3.VII.1995, C.P.; 1 spec., Cârțișoara, Vama Cucului (SB), 30.VII.1994, A.S.; 7 specs, Ieud (MM), 4-9.VII.1995, G.A., I.M.; 1 spec., Bârsana (MM), 5.VII.1995, C.P.; 8 specs, Repedea f.c. (MM), 29.VI.1997, A.S.; 1 spec., Smereceni clearing, 7 km towards the Repedea (MM), 22.VIII.1997, E.R.; 1 spec., Mara (MM), 26.VI.1998; 1 spec., Făina, Vaser Valley Forestry Railway (MM), 21.VII.2004, C.P. (Fig. 2).

Host plants: Salix sp. (Warchałowski, 2010).

\section{Chrysomela cuprea Fabricius, 1775}

Material: 1 spec., Buștenari, Telega (PH), without other data, coll. R.C.; 3 specs, București, without other data, coll. R.C.; 1 spec., Mireșu Mare (MM), coll. E.V.; 2 specs, Stănița (NT), 7.V.1966; 1 spec., Eșelnița Valley (M'H), 17.VI.1970, A.R.; Putna Valley (SV), 3.-4.VIII.1971, coll. I.C. (2 specs), 26.V.1980, coll. I.C. (2 specs); 2 specs, Metalici (Metaliferi) Mts (HD), 2.VII.1980, G.A.; 1 spec., Strâmtura (MM), 3.VII.1995, C.P.; 1 spec., Vadu Izei (MM), 9.VII.1995, I.M. (Fig. 3).

Host plants: Salix sp. (Mohr, 1966).

Chrysomela lapponica Linnaeus, 1758

Material: 1 spec., Transylvania, without other data, coll. D.K. (Fig. 4).

Host plants: Betula sp., Salix sp., Populus sp. (Mohr, 1966).

\section{Chrysomela populi Linnaeus, 1758}

Material: 10 specs, Buștenari, Telega (PH), without other data, coll. R.C.; 5 specs, Turda (Thörenburg) (CJ), without other data, coll. R.C.; 2 specs, Grâușorul (MS), without other data, coll. R.C.; 1 spec., Mireșu Mare (Nagynyires) (MM), 1942, coll. E.V.; 1 spec., Băile Pucioasa, Sântimbru (Büdösfürdö) (HR), 1952, coll. E.V.; Pitești, 4.V.1953, N.S. (1 spec.); 8 specs, Pasărea forest, Brănești (IF), 7.IV.1946, X.S.P.; 1 spec., Budești (CL), 16.VI.1947, X.S.P.; 4 specs, Voinești (DB), 23.V.1950, X.S.P.; Pitești (AG), 2.IV.1950, N.S. (2 specs), 9.V.1959, N.S, (3 specs), 9.V.1961 (2 


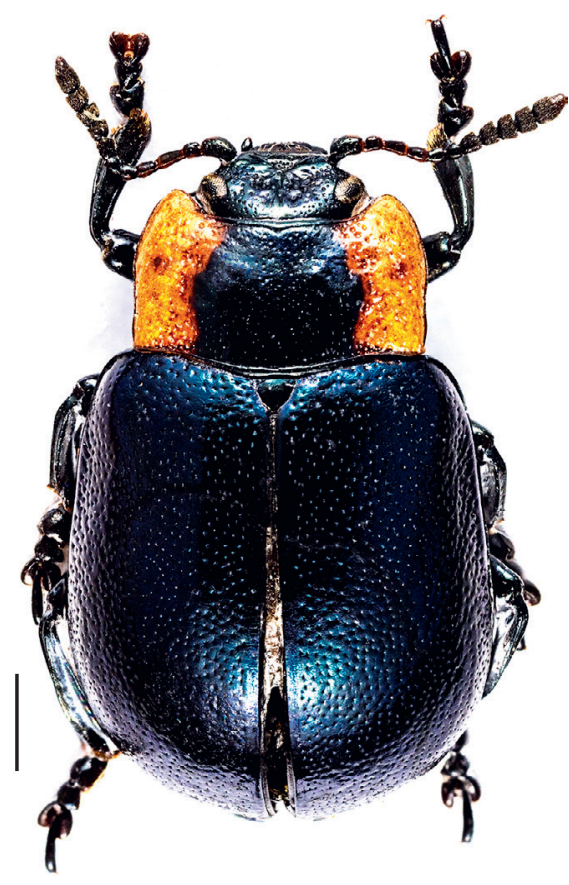

Fig. 2 - Chrysomela collaris. Scale bar $1 \mathrm{~mm}$.

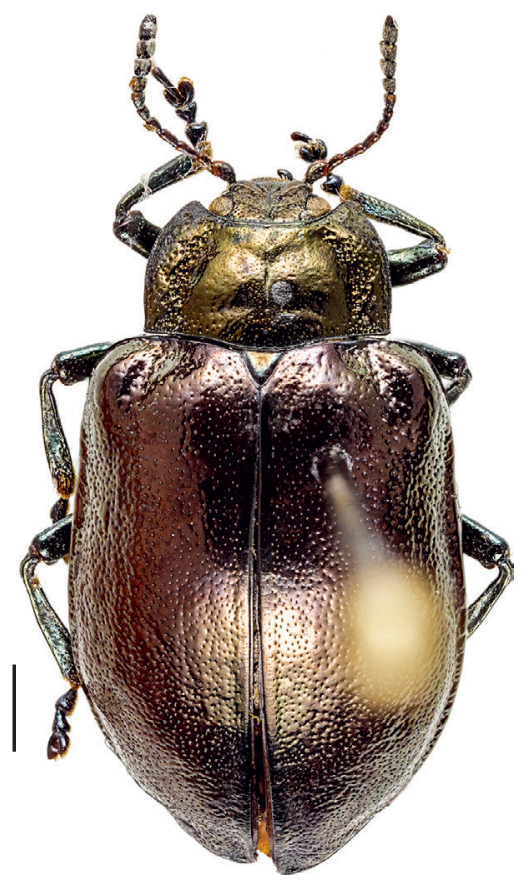

Fig. 3 -Chrysomela cuprea. Scale bar $1 \mathrm{~mm}$. 
specs), 24.IV.1970, N.S. (1 spec.); 1 spec., Băneasa forest (IF), 10.V.1952, N.S.; 2 specs, Braşov (BV), VII.1952, X.S.P.; 1 spec., Câmpulung Moldovenesc, Runc (SV), 24.V.1953, coll. I.C.; 2 specs, Vatra Dornei (SV), 30.VII.1953; 3 specs, Andronache forest, București, 12.VI.1954, A.R.; 1 spec., Petroșani (HD), 25.VI.1954, V.I.R.; Comana forest (GR), 8-9.V.1958, N.S. (15 specs), 20.VIII.1970, A.R. (2 specs), 7.VI.2006, R.S. (3 specs); 4 specs, Jegălia (CL), 19.VI.-30.VII.1955, coll. I.C.; 1 spec., Breasta, Craiova (DJ), 21.VII.1956, X.S.P.; 1 spec., Șimleu Silvaniei (SJ), 29.VII.1956, X.S.P.; 4 specs, Târgoviște (DB), 23.VIII.1957, A.R.; 2 specs, Voinești (DB), 23.VIII.1957, A.R.; București, 18,VI.1958, A.R. (2 specs), 15.IV.1963, A.R. (1 spec.), 20.V.1980, M.T. (1 spec.); 1 spec., Suceava (SV), 12.V.1959, I.N.; 1 spec., Mogoșoaia forest (IF), 26.V.1959, X.S.P.; 1 spec., Căldărușani (IF), 30.V.1959, A.R.; 1 spec., Cetate (DJ), 5.V.1960; 9 specs, Ciovârnășani (MH), 24.VI.1961, N.S.; 1 spec., Dumbrava forest, Sibiu (SB), 29.VI.1961, A.P.G.; 1 spec., Pufești (VN), 4.V.1962; 1 spec., Rarău Mts, Eastern Carpathians (SV), 7.VII.1962; 3 specs, Bozovici (CS), 6.VII.1962, I.D.; Vidra (VN), 17-18.VII.1962, X.S.P., A.R. (4 specs), 19.VII.1963, A.R. (2 specs); 1 spec., Săcele (BV), 2.IX.1962, X.S.P.; 2 specs, Gârla Mare (MH), 24.IV.1964; 1 spec., Vaideeni (VL), 10.VI.1964, M.V.; Herculane Baths (Băile Herculane) (CS), 29.VII.1964, N.S. (3 specs), 23.VII.1965, N.S. (3 specs); 2.IX.1991, C.P. (1 spec.); 1 spec., Mraconia (MH), 23.V.1967, Șt.N.; 7 specs, Valea Lungă, Cricov (DB), 28.V.- 5.VII.1967, A.R.; 9 specs, Curtea de Argeș (AG), 21.VI.1967, V.A.; 5 specs, Gorgota (PH), 4.VII.196720.IV.1968, A.R., X.S.P.; 1 spec., Bașta (NT), 16.IV.1968; 1 spec., Eșelnița Valley (MH), 1.VI.1968, X.S.P.; 10 specs, Örșova (MH), 29.VI.1969- 18.VI.1970, Şt.N., A.R.; 9 specs, Eșelnița (MH), 15-16.VI.1970, X.S.P., A.R.; 3 specs, Ogradena (MH), 21.VI.1970, A.R.; 1 spec., Șandru (BC), 8.VI.1971, M.W.; 1 spec., Dofteana (BC), 11.VI.1971, M.W.; 3 specs, S̉lănic Moldova (BC), 11.VI.1971; 1 spec., Moldova Nouă (CS), 22.V.1972, N.S.; 1 spec., Piatra Arsă, Bucegi Mts (PH), 22.VI.1972, M.W.; 2 specs, Crevedia Mare (GR0, 17.VIII.1976, M.A.; 1 spec., Predeal (BV), 31.VII.1977; 1 spec., Frunzaru forest, Sprâncenata (OT), 30.VIII.1980, G.A.; 1 spec., Buru, Trascău Mts (CJ), 30.IV.1980, M.A.; 4 specs, Sărata Monteoru (BZ), 19.VII.1981, V.B.; Gura Motrului (MH), 30.VII.1981, I.M. (1 spec.), 21-22.VI.1983, R.S., I.M. (9 specs); 1 spec., Strehaia (MH), 17.V.1982, D.D.; 14 specs, Slătioara Secular Forest (SV), V.1983, M.B.; 12 specs, Arginești forest, Gura Motrului (MH), 23.VI.1983, R.S., C.P.; 1 spec., Vânju Mare (MH), 15.VII.1983, I.M.; 164 specs, Foieni (SM), 14-15.VI.1985, R.S., I.M., C.P., N.G.; 2 specs, Livada (SM), 12.VI.1985, I.M.; 2 specs, Turulung Vii (SM), 13.VI.1985, R.S.; 6 specs, Moftin (SM0, 1.VII.1985, M.A.; 2 specs, Sâmbăta de Sus (SB), 4.VII.1985, N.G.; 1 spec., Poiana Neamțului (SB), 19.VII.1985, I.Ca.; 1 spec., Resighea (SM), 25.IV.1986, C.P.; 1 spec., Șelătruc f.c., Gutăi Mts (SM), 16.V.1986, R.S.; 1 spec., Măguri-Răcătău (CJ), 21.IX.1986, M.M.; 2 specs, Cămârzana (SM), 19.V.1987, C.P.; 1 spec., Călinești Oaș (SM), 21.V.1987, R.S.; 1 spec., Negureni, Valea Cișmelelor (CT), 18.V.1993, C.H.; 13 specs, Ieud (MM), 4.VII.-8.IX. 1995, P.C., G.A., I.M.; 2 specs, Ieud (MM), 4.VII.1995, I.M.; 8 specs, Slătioara f.c., Strâmtura (MM), 7.VII.-7.IX.1995, P.C., G.A., Mi.S., I.M.; 1 spec., Celic Dere Monastery (TL), 20.V.1997, C.P.; 1 spec., Colnic f.c., 2 km upstream from Mara, Desești (MM), 17.VII. 1998, A.V.; 1 spec., Nucet (DB), 19.X.1998, M.S.P.; 1 spec., Gârliște (CS), 7.VII.2001, A.S.; 7 specs, Leordina (MM), 15.VI.2003, R.S., C.P.; 2 specs, Coșnea f.c., Poienile de sub Munte (MM), 16.VI.2003, C.P.; 1 spec., Repedea f.c., Repedea (MM), 18.VI.2003, R.S.; 2 specs, Baicu f.c., Dragomirești (MM), 21.VI.2003, C.P., D.S.; 1 spec., Cetățuia, Găujani (GR) (Danube River at kilometer 518), 26.IV.2004, M.S.; 2 specs, Pietroșani (Danube River at kilometer 519) (GR), 28.IV.2004, M.S.; 6 specs, 1 spec., the Cama 
islet (the Danube River at kilometer 510) (GR), 29.IV.2004, M.S.; 9 specs, Penciu forest, Braniștea (Danube River at kilometer 480) (GR), 22.VI.2004, C.P.; 6 specs, Ochiul Boului Island (Danube River at kilometer 451) (GR), 23.VI.2004, D.M.; 1 spec., Măgura (BZ), 23.VI.2004, V.U.; 1 spec., Făina, Vaser Valley Forestry Railway (MM), 22.VII.2004, M.S.; 1 spec., Socolău forest, Poienile de sub Munte (MM), 23.VII.2004, C.B.; 1 spec., the confluence of the Tisza and Viseu rivers (MM), 24.VII.2004, R.S.; 2 specs, Sărata (SB), 27.VI.-2005-20.VIII.2008, C.B.; 10 specs, Crucea de Piatră (GR), 7.VI.2006, R.S.; 1 spec., Bârnova forest (IS), 26.V.2011, I.Șt.I.; 5 specs, Durău (NT), 1.VII.2011, I.Șt.I.; 19 specs, Gârla Mare (MH), 17.V.2015, G.C.; 1 spec., Sadu Valley (SB), 20.V.2015, C.C.; 1 spec., Sânpetru German (AR), 27.V.2015, E.I.; 2 specs, Șuvara Sașilor (SB), 27.VII.2015, C.C; 1 spec., France, Besançon, without other data, coll. A.L.M.

Host plants: Salicaceae (Populus alba, P. tremula) (Warchałowski, 2010).

Chrysomela saliceti saliceti Weise, 1884

Material: 1 spec., Mireşu Mare (Nagynyires) (MM), 1942, coll. E.V.; 4 specs, Herculane Baths (Băile Herculane) (CS), whitout other data, coll. N.S.; 1 spec., whitout other data, coll. I.C.; 1 spec., Ardeluţa, Tarcău (NT), 19.V.1949, coll. I.C.; 1 spec., Grienţieş (NT), 20.V.1949, coll. I.C.; 1 spec., Pasărea forest (IF), 29.III.1951, N.S.; Jegălia (CL), 25.V.1955, coll. I.C. (2 specs), 19-24.VI.1955, coll. I.C. (3 specs), 14-30.VII.1955, coll. I.C. (3 specs); 2 specs, Simleu Silvaniei (SJ), 30.VII.1957, X.S.P.; 1 spec., Târgu-Jiu (GJ), 2.VI.1959; 14 specs., Cetate (DJ), 5.V.1960; 3 specs, Piteşti (AG), 9.V.1961, coll. N.S.; 5 specs, Ciutura (DJ), 15.VI.1963; 2 specs, Cărbuneşti (PH), 15.VII.1963; 1 spec., Craiova (DJ), 5.VI.1964, Şt.N.; 2 specs, Moşna (IS), 12.IX.1968; 1 spec., Orşova (MH), 29.VI.1969, Şt.N.; 1 spec., Eşelniţa (MH), 29.III.1970, Şt.N.; 2 specs, Ogradena (MH), 21.VI.1970, A.R.; 1 spec., Slănic Moldova (BC),7.VI.1971; 7 specs, Răstoaca (VN), 2.IX:1971, coll. N.S.; 1 spec., Sfântu-Gheorghe, Danube Delta (TL), 8.VII.1972; 1 spec., Văratec Monastery (NT), 12.VIII.1976, R.S.; 2 specs, Valley of Azuga River (PH), 1.VIII.1996, C.H.; 4 specs, Floreşti (GR), 24.V.1997, C.P.; 1 spec., Mara (MM), 26.VI.1998; 2 specs, the confluence of the Pop Ivan and Hututeanca rivers (MM), 18.VII.1994, G.C.; 1 spec., Lereşti (AG), 19.VII.2003, Mi.S.; 1 spec., Izvorul Dulce, Beceni (BZ), 29.VII.2003, V.U.; 1 spec., Balauru Hill, Beceni (BZ), 15.VI.2004, V.U.; 2 specs, Făina, Vaser Valley (MM), 21.VII.2004, R.S.; 13 specs, Valley of the Socolău River, $2 \mathrm{~km}$ upstream of the confluence of the Socolău and Rica rivers (MM), 23.VII.2004, R.S.; 2 specs, Crucea de Piatră (GR), 7.VI.2006, R.S. Host plants: Salicaceae (Salix purpurea, S. viminalis) (Warchałowski, 2010).

\section{Chrysomela tremula tremula Fabricius, 1787}

Material: 1 spec., whitout other data, coll. I.C.; 1 spec., Bucovăţ(DJ), VIII.1951, X.S.P.; 1 spec., Jegălia (CL), 19.VI.1955, coll. I.C.; 1 spec., Tufa Valley, Sinaia (PH), 8.VII.1958, A.R.; 1 spec., Poiana Ţapului (PH), 10.VII.1958, A.R.; 1 spec., Burdujeni forest (SV), 12.VI.1959, I.N.; 1 spec., Dumbrava forest (SB), 29.VI.1961, A.P.G.; 3 specs, Cumpătu, Sinaia (PH), 29.VI.1969, A.R.; 1 spec., Perchiu (BC), 12.VI.1971; 1 spec., Sinaia (PH), 19.VII.1972; 1 spec., Socolău forest, Poienile de sub Munte (MM), 19.07.2004, C.B.; 1 spec., Frumoasa Valley (SB), 6.VIII.2014, C.C.

Host plants: Salicaceae (Populus alba, P. tremula) (Warchałowski, 2010). 
Chrysomela vigintipunctata vigintipunctata (Scopoli, 1763)

Material: 2 specs, Buștenari, Telega (PH), without other data, coll. R.C.; 2 specs, Mireșu Mare (Nagynyires) (MM), 1942, coll. E.V.; 1 spec., Tarcău (NT), 16.VI.1949, coll. I.C.; 2 specs, Pasărea forest (IF), 7.IV.1951, N.S.; 1 spec., Băile Pucioasa, Sântimbru (Büdösfürdö) (HR), 1952, coll. E.V.; 5 specs, București, V.1956; 1 spec., Tufa Valley, Sinaia (PH), 8.VII.1958; 1 spec., Gâdinți (NT), 2.VII.1962, coll. I.C.; 1 spec., Periprava, Danube Delta (TL), 11.VII.1962, M.W.; 1 spec., Stănița (NT), 7.V.1966, coll. I.C.; 1 spec., Cheile Turzii (CJ), 4.VI.1966, D.L.; 1 spec., Slătioara Secular Forest (SV), 18.V.1969, M.B.; 1 spec., Strehaia (MH), 10.VI.1982. R.S.; 1 spec., Cămârzana (SM), 19.V.1987, R.S.; 3 specs, Tinosu forest (PH), 10.VI.1987. V.G.; 1 spec., Plopiș Mts, Aleșd (BH), 25.VI.1994, A.S.; 1 spec., Strâmtura, Slătioara River (Berşotă rivulet) (MM), 3.VII.1995, C.P.; 1 spec., Izvorul lui Dragoş, Moisei (MM), 13.VII.1996, C.P.; 1 spec., Florești (GR), 24.V.1997, C.P.; 2 specs, Valley of Frumuşeaua River, 7 km upstream Crasna Vişeului (MM), 23-25.VI.1997, R.S., A.S.; 2 specs, Repedea f.c., Repedea (MM), 23-26.VI.1997, I.M., C.P.; 2 specs, Mara (MM), 26.VI.1998; 1 spec., Bârsa Tămașului, The National Park "Piatra Craiului" (BV), 9.VI.2002, C.P.; 2 specs, Pietroșani, (the Danube River at kilometer 521) (GR), 28.IV.2004, M.S.; 1 spec., the islet Cama (the Danube River at kilometer 510) (GR), 29.IV.2004, M.S.; 10 specs, Onceoaia Valley, Sătic, The National Park "Piatra Craiului" (AG), 23.V.2004, R.S.; 25 specs, Bârsa Valley, 2 km downstream Plaiul Foii, The National Park "Piatra Craiului" (BV), 24.V.2004, R.S., C.B.; 2 specs, the Elena Islet (the Danube River at kilometer 474), Gostinu (GR), 22.VI.2004, C.P.; 6 specs, Penciu forest (GR), 22.VI.2004, C.P.; 1 spec., "Gurile Argeșului" (Argeș River flows into the Danube at Oltenița) (CL), 23.VI.2004, C.P.; 1 spec., Dâmbovicioara Gorges, The National Park "Piatra Craiului" (AG), 21.VI.2005, R.S.; 6 specs, Frăsinet, Valea Ierii (CJ), 19.VI.2012, M.S.; Sadu Valley (SB), 20.V.2015, C.C., L.M. (6 specs), 17.VI.2015, Mi.S. (1 spec.); 2 specs, Dobra Valley (SB), 3.VI.2015, C.A., G.C.; 7 specs, Podeni (MH), 10.VI.2015, G.C.; 2 specs, Micăsasa (SB), 18-19.VI.2017, C.C., Vi.G; 1 spec., Serbia, Lazareva River canyon, Kučaj Mts, 1.VI.1996, V.G.

Host plants: Salicaceae (Salix sp.) (Warchałowski, 2010).

\section{Plagiodera Chevrolat, 1836}

Plagiodera versicolora (Laicharting, 1781)

Material: 1 spec., Mogoșoaia forest(IF), 26.V.1959, A.R.; 1 spec., Chilia Veche, Danube Delta (TL), 7.V.1964, X.S.P.; 28.V.1967, A.R.; 2 specs, Gorgota (PH), 4.VII.1967, A.R.; 2 specs, Valea Lungă, Cricov (DB), 5.VII.1967, A.R.; 6 specs, Eșelnița (MH), 11.VIII.1969-16.VI.1970, X.S.P., A.R.; 1 spec., Ogradena (MH), 12.VIII.1969, X.S.P.; 7 specs, 21.VI.1970, A.R.; 13 specs, Orșova (MH), 1.X.1969-18.VI.1970, A.R.; 2 specs, Snagov forest (IF), 12.X.1979, R.S.; 1 spec., Nanov forest (TR), 13.V.1980, R.S.; 1 spec., Metalici Mts (HR), 2.VII.1980, G.A.; 48 specs, Afumați (IF), 9.IV.1981, C.P.; 41 specs, Dunavățu (TL), 31.V.-9.VI.1994, C.H.; 2 specs, Plopiș Mts, Aleșd (BH), 25.VI.1994, A.S.; 3 specs, Strâmtura (MM), 3.VII.1995, C.P.; 3 specs, Ieud (MM), 4.VII.1995, C.P.; 6 specs, Ieud ("Păcuri”), 8.VII.1995, leg. G.A.; 4 specs, Bârsana (MM), 5.VII.1995, C.P.; 4 specs, Vadu Izei (MM), 9.VII.1995, I.M.; 1 spec., Celic Dere Monastery (TL), 24.V.1997, R.S.; 4 specs, Valley of the Budac River, Simionești (BN), 28.VI.1997, D.R.; 1 spec., Săcel (MM), VI.1998; 2 specs, Tătaru dam, Mara River, Ocna Şugatag (MM), 16-19.VII.1998, M.S.P.; 5 specs, Mara (MM), 21.VII.1998, I.M.; 1 spec., Izvoru Dulce, Beceni (BZ), 7.VII.2002, V.U.; 13 specs, Leordina (MM), 15.VI.2003, C.P., Mi.S.; 1 spec., Coşnea f.c., Poienile de sub Munte (MM), 16.VI.2003, C.P.; 1 spec., 
Repedea f.c., Repedea (MM), 18.VI.2003, C.P.; 1 spec., Copănița island (Danube River at kilometer 696) (DJ), 17.V.2004, C.P.; 1 spec., Măgura (BZ), 23.VI.2004, V.U.; 1 spec., Făina, Vaser Valley (MM), 21.VII.2004; 3 specs, Valley of the Socolău River, 2 $\mathrm{km}$ upstream of the confluence of the Socolău and Rica rivers (MM), 19-23.VII.2004, R.S., C.B.; 15 specs, the confluence of the Vișeu and Tisa rivers (MM), 24.VII.2004, R.S.; 2 specs, Făgăraș Mts, Valley of Buda River (AG), 4.VIII.2004, R.S; 1 spec., Germany, Burgberg im Allgäu, Bavaria (whitout other data).

Host plants: Salicaceae (Salix sp., Populus sp.) (Warchałowski, 2010).

\section{Plagiosterna Motschulsky, 1860 \\ Plagiosterna aenea aenea (Linnaeus, 1758)}

Material: 1 spec., Ardeluţa, Tarcău (NT), 24.V.1949, coll. I.C.; 1 spec., Grințieș (NT), 30.V.1949, coll. I.C.; 1 spec., Sibișel (Beriu) (HD), VI.1950, I.L.; 1 spec., Agigea (CT), 31.VII.1957, X.S.P.; 1 spec., Broșteni (SV), V.1958, coll. I.C.; 1 spec., Tufa Valley, Sinaia (PH), 8.VII.1958, A.R.; 1 spec., Barnar, Crucea f.c., Bistrița Mts. (SV), 1.VIII.1958, coll. I.C.; 1 spec., Gemenea, Voinești (DB), 19.V.1961, A.R.; Rarău Mts, Eastern Carpathians (SV), VII.1962 (1 spec.), 10.VI.1992, I.C. (2 specs); 3 specs, Bălătău (Tarnița) Lake, Nemira Mts, Sălătruc (BC), 4-8.VII.1965 (2 specs), 10.VI.1971 (1 spec.); Lerești (AG), 12.V.1966, I.D. (4 specs), 20.VII.2003, M.S. (7 specs); 1 spec., Padiș, Bihor Mts (BH), 1.VII.1978, G.A.; 1 spec., Călugăreni (GR), 6.VII.1978; Poiana Neamțului (SB), 16.VII.-19.VIII.1985, R.S., I.M., I.D. (58 specs), 15.V.1996, I.D. (8 specs); 9 specs, Turnu Roșu (SB), 13.V.1986, I.D.; 3 specs, Covasna (CV), 20-24.VI.1987, V.B.; 5 specs, Valea Glodului, Leaota Mts, 4.V.1990, N.R.; 1 spec., Dâmbovicioara Gorges, 15.V.1990, G.A.; 2 specs, Comandău (CV), 11-27. VI.1992, I.C.; 1 spec., Canaraua Fetii (CT), 20.V.1993, C.P.; 3 specs, Cârțișoara, Vama Cucului (SB), 30.VII.1994, A.S.; 1 spec., Sinaia, Cumpătu (PH), 27.V.1994, coll. I.C.; 1 spec., Caraiman chalet, Bucegi Mts (PH), 11.VI.1995, D.M.; Piatra Craiului Mts, Sătic, Valley of Dâmbovița River (AG), 22.VI.1995, L.O.P. (3 specs), 22.VI.2005, M.S., C.B., I.M., E.I., M.St. (41 specs); 1 spec., Strâmtura (MM), 3.VII.1995, C.P.; 19 specs, Strâmtura, Slătioara River, Berșotă stream (MM), 3.VII.1995, C.P., I.M.; 17 specs, Bârsana (MM), 5.VII.1995, C.P.; 5 specs, Izvorul Izei (MM), 6.VII.1995, C.P.; 4 specs, Slătioara f.c., Strâmtura (MM), 12.VII.-7.IX.1995, G.A., I.M.; 1 spec., Moisei, Izvorul lui Dragoș f.c. (MM), 13.VII.1995, C.P.; 1 spec., Săpânța, Nireș stream, Șipote cascade (MM), 16.V. 1996, Mi.S.; 3 specs, Valley of Săpânța River, upstream Săpânța (MM), 18.V.1996, Mi.S., D.R.; 5 specs, Valley of Săpâncioara River, Igniș plateau (MM), 20.V.1996, D.R., Mi.S.; 1 spec., Călinești, Săcătura f.c. (MM), 7.VII.1996, I.M.; 38 specs, Smereceni clearing, 7 km upstream Repedea (MM), 22-27.VI.1997, R.S., I.M.; 40 specs, Repedea, Repedea f.c. (MM), 22-29.VI.1997, R.S., I.M., A.S., C.P.; 11 specs, Valley of Frumușeaua River, 7 km upstream Crasna Vișeului (MM), 29.VIII.1997, A.S., R.S.; 1 spec., Izvorul Albastru al Izei (MM), 17.VI.1998; 1 spec., Runcu dam, upstream Tătaru Gorges (MM), 17.VI.1998; 2 specs, Colnic f.c., 2 km upstream Mara, Desești (MM), 17.VI1.1998, I.M.; 2 specs, Valley of Mara River, 2 km upstream Mara, Desești, 18.VII. 1998, I.M.; 1 spec., 1 km upstream Mara (MM), 21.VI1.1998, I.M.; 7 specs, Valley of Runcu River, Stedea f.c., Desești (MM), 29.VII. 1998, M.S.P.; 1 spec., Jepii Mici, Bucegi Mts, Bușteni (PH), 9.VIII.1998, M.S.P.; 2 specs, Tismana (GJ), 29.VII.1999, G.A.; 29 specs, Piatra Craiului Mts, Bârsa Groșetului, 4 km upstream Plaiul Foii chalet (BV), 9.VI.2000, C.P.; 7 specs, Piatra Craiului Mts, Valley of Diana rivulet, 10.VI.2000, C.P.; 1 spec., Piatra Craiului Mts, Bârsa Valley (AG), 4.VII.2000, L.O.P.; 2 specs, Piatra Craiului Mts, Scorobia Valley (AG), 5.VII.2000, L.O.P., A.V.; 
Piatra Craiului Mts, Gura Bârsei (BV), 10.VI.2000, C.P. (2 specs), 14.VI.2001, C.P. (30 specs), 1-2.VII.2002, C.P. (93 specs); 2 specs, Piatra Craiului Mts, Sătic, Scorobia Valley (AG), 5.VII.2000, A.S.; 11 specs, Piatra Craiului Mts, "Valea Scheilor" chalet (AG), 5.VII.2000, A.S.; Piatra Craiului Mts, Zăpodia Valley, Sătic (AG), 5.VII.2000, C.P. (1 spec.), 28.VI.2002, C.P. (4 specs); 10 specs, Piatra Craiului Mts, Sătic, Speriata Valley (AG), 6.VII.2000, A.S., C.P.; 15 specs, Piatra Craiului Mts, Dragoslăveni Valley, 8.VII.2000, C.P., A.V.; Piatra Craiului Mts, Valley of the Bârsa Fierului River (BV), 12.V.2001, C.P. (4 specs), 24.V.2004, R.S., C.B., C.P. (11 specs); 1 spec., Nera Gorges, Lindeni clearing (CS), 15.V.2002, M.S.; 1 spec., Piatra Craiului Mts, Valea lui Ivan, 27.VI.2002, C.P.; 66 specs, Piatra Craiului Mts, Berila Valley, Sătic (AG), 30.VI.2002, C.P.; Piatra Craiului Mts, Oncioaia Valley, Sătic (AG), 30.VI.2002, R.S. (47 specs), 3.VII.2002, C.P. (2 specs), 22-23.V.2004, R.S., C.P. (93 specs); 3 specs, Poienile de sub Munte, $500 \mathrm{~m}$ downstream the confluence of the rivers Rica and Budescu (MM), 14.VI.2003, C.P., R.S.; Făina, Valley of Vaser River (MM), 19.VI.2003, R.S., M.S., C.P. (83 specs), 21.VII.2004, M.S., C.P. (4 specs); 2 specs, Țibleș Mts, Lunca lui Cosiță, 4 km upstream Baicu f.c., Dragomirești (MM), 22.VI.2003, C.P.; 34 specs, Piatra Craiului Mts, Măgura (BV), 21.V.2004, R.S., C.P., C.B.; 120 specs, Piatra Craiului Mts, Valley of the Bârsa Mare River, $2 \mathrm{~km}$ downstream of Plaiul Foii chalet (BV), 24.V.2004, R.S., C.B.; 6 specs, Bucegi Mts, Predeal (BV), 5.VII.2004, V.U.; 3 specs, Bucegi Mts, Sinaia, Furnica Peak (PH), 7.VII.2004, V.U.; 3 specs, the confluence of the Pop Ivan and Hututeanca rivers (MM), 18.VII.2004, G.C., C.B.; 2 specs, Valley of Socolău River, Poienile de sub Munte (MM), 19.VII.2004, G.C.; 2 specs, Maramureș Mts, Făina, Valley of Vaser River (MM), 21.VII.2004, R.S.; 29 specs, Maramureș Mts, Valley of Făina River (MM), 22.VII.2004, R.S., M.S.; 1 spec., Maramureș Mts, Valley of Vaser River (MM), 22.VII.2004, C.P.; 4 specs, Valley of the Vaser River, Făina-Bardău transect (MM), 22.VII.2004, C.B.; 5 specs, the confluence of the Vișeu and Tisa rivers (MM), 24.VII.2004, R.S., C.P.; 1 spec., Poienile de sub Munte (MM), 25.VII.2004, R.S.; 58 specs, Făgăraș Mts, Valley of Buda River (AG), 4.VIII.2004, R.S., M.S., A.P.; 75 specs, Făgăraș Mts, Vidraru Lake, 5 km away from Cumpăna chalet (AG), 4.VIII.2004, R.S.; 17 specs, Făgăraș Mts, Valley of Otic River (AG), 4.VIII.2004, R.S.; 146 specs, Făgăraș Mts, Vidraru Lake, "Valea cu Pești" chalet (AG), 6.VIII.2004, M.S., R.S.; 43 specs, Făgăraș Mts, Vidraru Lake, Stan Valley (AG), 7.VIII.2004, R.S.; 13 specs, Făgăraș Mts, Vidraru Lake, Moliviș (AG), 7.VIII.2004, M.S.; 16 specs, Făgăraș Mts, Vidraru Lake, Curmătura Valley (AG̉), 8.VIII.2004, R.S.; 17 specs, Făgăraș Mts, Vidraru Lake, Cumpăna - Clăbucet Mt. route (AG), 8.VIII.2004, M.S.; 6 specs, Piatra Craiului Mts, Dâmbovicioara Gorges (AG), 9.VIII.2004, M.S.; 4 specs, Pasărea forest (IF), 5.VI.2005, C.P.; 22 specs, Piatra Craiului Mts, Sătic (AG), 22.VI.2005, R.S., M.S., E.I.; 1 spec., Sărata (SB), 4.V.2007, C.B.; 2 specs, the Natural Reserve Tișița Gorges (Cheile Tișiței), Vrancea Mts (VN), 21.V.2013, M.S.; Putna Vrancea Natural Park, Lepșa (VN), 21.V.2013, M.S. (3 specs), 31.V.2015, A.K., A.L. ( 2 specs); 7 specs, Putna - Vrancea Natural Park, Valley of Băhneanu rivulet (VN), 29.VI.2013, C.C.; 3 specs, Putna - Vrancea Natural Park, Soveja, Valley of Cremenet River (VN), 19.V.2014, C.C.; Voineasa, Jidoaia Valley (VL), 21.V.2014, E.I. (1 spec.), 4.IX.2014, C.C. (1 spec.), 21.V.2015, M.S., L.M. (2 specs); Lotrioara Valley (SB), 1-4. VII.2014, C.C., E.I. (38 specs), 5-17.VIII.2014, C.C. (4 specs); 3 specs, Tălmăcel, Râușor Valley (SB), 1-2.VII.2014, C.C.; 2 specs, Valley of Sebeş River (between Oaşa Lake and Şugag) (AB), 11.VII.2014, C.C.; 7 specs, Sadu - Râu Sadului, Sadu Valley (SB), 20.V.2015, Mi.S., C.C., L.M.; spec., Postăvaru, Poiana Brașov (BV), 13.V.2015, M.S.; 3 specs, Sădurel Valley (SB), 20.V.2015, Mi.S., E.I.; 4 specs, Putna - Vrancea 
Natural Park, Valley of Strâmba stream (VN), 30.V.2014, A.K.; 5 specs, Sadu Valley (SB), 20.V.2015, M.S.; 2 specs, Voineşiţa Valley (VL), 21.V.2015, Mi.S., E.I.; 4 specs, Dobra Valley (AB), 3.VI.2015, C.A.; 4 specs, Tău Bistra (AB), 19.VI.2015, C.C.; 14 specs, Retezat National Park, The Gemenea Scientific Reserve (HD), 3.VII.2015, C.A., G.C.; 1 spec., Brăduleț, Vâlsan Valley (AG), 29.VII.2015, M.S.; 3 specs, Paltin Valley (SB), 3.VIII.2015, M.S.; 1 spec., Crasna Monastery (PH), 14.V.2016, Du.S.

Host plants: Betulaceae (Alnus sp.) (Mohr, 1966).

Subtribe Gastrophysina Kippenberg, 2010

Gastrophysa Chevrolat, 1836

Gastrophysa (s.str.) polygoni polygoni (Linnaeus, 1758)

Material: 1 spec., Măcin (TL), without other data, coll. A.L.M.; Mireşu Mare (Nagynyires) (MM), 1940 (1 spec.), 1941 (3 specs), 1942 (2 specs), coll. E.V.; 1 spec., Hanu Conachi (GL), VII.1950, coll. I.C.; 2 specs, Câmpulung Moldovenesc (SV), 19.IV.1951 - 7.V.1952, coll. I.C.; Pasărea forest (IF), 8-28.IV.1951, N.S. (2 specs), 19.VI.1965, A.R. (1 spec.); Comana forest (GR), 5.V.1951, N.S. (1 spec.), 2223.VII.1963, Șt.N. (2 specs), 18.IX.1964, A.R. (3 specs); București, 8.IV.1954, X.S.P. (1 spec.), 3.IV.1963, A.R. (1 spec.), 8-23.IV.1971, Ct.C. (5 specs), 6.VI.1971, A.R. (1 spec.); 4 specs, Negoiești (DJ), 25.IV.1954; 7 specs, Armășești (IL), 27.IV.1954; 2 specs, Rădulești (Brazi) (IL), 27.VI.1954; 1 spec., Agigea (CT), 21.VIII.1954; 2 specs, Căldărușani forest (IF), 9.VII.1955; 5 specs, Dăbuleni (DJ), 10.V.1956; 1 spec., Oltina (CT), 26.VI.1956, N.S.; 2 specs, Țigănești (Ciolpani) (IF), 4.V.1957, X.S.P.; 1 spec., Copăceni (IF), 17.VI.1958, A.R.; 2 specs, Scheia forest (SV), 8-12.V.1959, I.N.; 3 specs, Podari (DJ), 5.VI.1960, Șt.N.; Periprava, Danube Delta (TL), 12.VII.1962, M.W. (1 spec.), 2.VII.1963, X.S.P., (1 spec.), 9.V.1964, X.S.P. (4 specs), 26.IV.1966, Șt.N. (1 spec.), 16.VII.1966, X.S.P. (2 specs), 15.X.1966, Șt.N. (13 specs); 1 spec., Târnava forest (SB), 5.VI.1963; 1 spec., Tulcea (TL), 22.VI.1963, X.S.P.; Chilia Veche, Danube Delta (TL), 7.V.1964, M.W., I.S. (4 specs), 15.IV.1966, Șt.N. (1 spec.); Andronache forest, București, 29.IV.1965, A.R. (1 spec.), 20.XI.1966, A.R. (1 spec.); 1 spec., Sinaia (Cumpătu), 22.V.1965, Șt.N.; Hagieni forest (CT), 7.VI.1965, Șt.N. (26 specs), 21.VII.1997, C.P. (2 specs); 1 spec., C.A. Rosetti, Danube Delta (TL), 6.X.1965, X.S.P.; 2 specs, Voinești (DB), 8.VIII.1966, X.S.P.; 3 specs, Nanov (TR), 2.X.1966, X.S.P.; Eșelnița (MH), 8.VI.1967, I.D. (1 spec.), 13-25.VIII.1969, X.S.P. (5 specs), 15-19.VI.1970, X.S.P. (12 specs); 2 specs, Cazanele Dunării (Danube Boilers) (MH), 10.VI.1967, Șt.N.; 1 spec., Niculițel (TL), 27.VI.1967, M.V.; 4 specs, Fundulea (IF), 29.VI.1967, A.R.; 2 specs, Codru Istrița (BZ), 14.IX.1967, X.S.P.; Caraorman, Danube Delta (TL), 5.X.1968, St.N. (1 spec.), 4.VIII.2006, E.I. (1 spec.); Orșova (MH), 28.X.1968, Șt.N. (1 spec.), 18.VI.1970, A.R. (5 specs); 4 specs, Ogradena (MH), 21.VI.1970, A.R.; 1 spec., Tușnad Baths (Băile Tușnad) (HR), 7.VIII.1970, X.S.P.; 1 spec., Bârlad (VS), 19.IX.1972, A.R.; Băneasa forest (București), 13.VII.1973, A.R. (1 spec.), 10.VII.2006, E.I. (1 spec.); 1 spec., Cocoș Monastery (TL), 2.IX.1973, M.W.; 1 spec. Prundu (GR), 28.IV.1977, R.S.; 3 specs, Zăvestreni forest (TR), 1.IX.1977, R.S.; 1 spec., Căscioarele forest, Dealu (IF), 7.VI.1979, R.S.; 6 specs, Dumitrana (IF), 3.VIII.1979, R.S.; 3 specs, Snagov forest (IF), 12.X.1979, R.S.; 2 specs, Poieni (TR), 20.VIII.1980, I.M.; 2 specs, Bistreț (DJ), 25.VI.1981, R.S.; 1 spec., Târnava (DJ), 21.VI.1982, R.S.; 2 specs, Foieni (SM), 14.VI.1985-24.IV.1986, C.P.; 1 spec., Cărășeu (SM), 29.VII.1985, I.D.; 9 specs, Sihlea (VN), 23.VI.1987, I.M., C.P.; 8 specs, Spătaru forest, Costești (BZ), 24.VI.1987, C.P., I.M.; 1 spec., Giulești (IF), 8.VII.1991; 4 specs, Maliuc, Mila 26 (TL), 8.VIII.1991, I.M.; 8 specs, Canaraua Fetii (CT), 20.V.1993 - 21.VI.1995, C.P.; 1 spec., Gârliște (CS), 
VI.1994. coll. I.C.; 2 specs, Plopiș Mts, Aleșd (BH), 25.VI.1994, A.S.; 1 spec., Celic Dere Monastery (TL), 24.V.1997, C.P.; 1 spec., Piatra Craiului Mts, Gura Bârsei (BV), 4.VII.2000, C.P.; 1 spec., Furnica Peak, Bucegi Mts (PH), 23.VI.2001, V.U.; 1 spec., The Muddy Volcanoes (Vulcanii Noroioși), Pâclele Mari (BZ), 18.IV.2002, V.U.; 1 spec., Izvoru Dulce, Beceni (BZ), 1.VI.2002, V.U.; 1 spec., Bârsa Tămașului. Piatra Craiului Mts, 9.VI.2002, C.P.; 1 spec., Valley of the Cvașnița River, Poienile de sub Munte (MM), 15.VI.2003, C.P.; 1 spec., Valley of the Pop Ivan River, $12 \mathrm{~km}$ upstream Crasna Vișeului (MM), 17.VI.2003, M.S.; 1 spec., the Natural Reserve "Marine Sand Dunes of Agigea" (CT), 17.VII.2003, A.P.; 1 spec., Cetățuia, Găujani (GR), (Danube River at kilometer 516), 26.IV.2004, M.S.; 1 spec., the Cama islet (the Danube River at kilometer 510) (GR), 29.IV.2004, M.S.; 1 spec., Lung islet (Ostrovul Lung) (the Danube River at kilometer km 470) (GR), 22.VI.2004, C.P.; 4 specs, Penciu forest, Braniștea (Danube River at kilometer 480) (GR), 23.VI.2004, M.S.; 2 specs, the Danube River at kilometer km 465 (GR), 24.VI.2004, C.P.; 1 spec., the confluence of the Pop Ivan and Hututeanca rivers (MM), 18.VII.2004, C.P.; 1 spec., Balauru Hill, West to Izvorul Dulce, Beceni (BZ), 4.V.2005, V.U.; 1 spec., Manafu forest, Izvoarele (GR), 22.VI.2006, R.S.; 13 specs, Moara Domnească (IF), 12.VI.2006, M.S.; 70 specs, Valea Gurbanului, Comana (GR), 11.VIII.2009, R.S., Iu.M.; 1 spec., Călimani National Park (SV), 20.VII.2012, I.Șt.I.; 11 specs, Turkey, Denizli Province, Koruçuk ("Focida" expedition), 3.08.2006, R.Z., S.G.; 4 specs, Turkey, Denizli Province, Buharkent ("Focida" expedition), 4.08.2006, C.P.; 1 spec., Syria, Al Assad Lake, 3.V.2010, V.G. ("Sabka" expedition).

Host plants: Polygonaceae (Rumex sp., Polygonum sp.) (Mohr, 1966).

\section{Gastrophysa viridula viridula (De Geer, 1775)}

Material: 1 spec., Transylvania, without other data, coll. D.K.; 2 specs, Comana forest (GR), 5.V.1951, N.S.; 3 specs, Lucina, Obcinele Bucovinei (SV), 3.VI.1951, coll. I.C.; 2 specs, Tecuci (GL), 5.VI.1954, N.S.; 16 specs, Beliș (CJ), 29.VII.1960, coll. I.C.; 1 spec., Beliş, Giurcuța de Sus, (CJ), 30.VII.1960, coll. I.C., 1 spec., Călimani Mts., 1500-1700 m a.s.1., 25.V.1963, coll. I.C.; 145 specs, Sinaia, Bucegi Mts (PH), 1,330 m a.s.l., 28-30.V.1964, I.D., A.R.; 152 specs, Poiana Stânii, Bucegi Mts (PH), 28.VI.1969, I.D.; 2 specs, Plopiș, Șișești (MM), 27.VI.1980, G.A.; 12 specs, Apuseni Mts, Onu Valley (?), 30.VI.1980; 4 specs, Diham chalet, Bucegi Mts (PH), 19.VII.1984, R.S.; 4 specs, Turulung VII (SM), 13.VI.1985, R.S.; 9 specs, Avrig (SB), 16.VII.1985, R.S., I.M.; Poiana Neamțului (SB), 17-20.VII.1985, R.S., I.Ca, I.M. (8 specs), 14 - 15.V.1986, I.M., I.D. (69 specs);12 specs, Cărășeu (SM), 20-30.VII.1985, I.D., V.G.; 5 specs, Călinești Oaș (SM), 31.VII.1985, C.P.; 36 specs, Sebeșu de Sus (SB), 12.V.1986, I.M.; 9 specs, Turnu Roșu (SB), 13.V.1986, I.M.; 7 specs, Şelătruc chalet, Gutâi Mts (SM), 15-16.V.1986, R.S., I.C.; Plaiul Foii, Piatra Craiului Mts (BV), 25-27.IV.1990, R.S. (5 specs), 28.V.-1.VI.1990, R.S. (27 specs), 12.VII.1990, I.M. (3 specs); Bârsa Groşetului, 4 km upstream Plaiul Foii, Piatra Craiului Mts (BV), 13.V.1990 (3 specs), 9.VI.2000 C.P. (15 specs); 3 specs, Valley of S,pirla River, Piatra Craiului Mts (BV), 30.V.1990, N.G.; 15 specs, Măgura Răcătău (CJ), 4.VI.1992, N.G.; 1 spec., Sohodol Gorges, Runcu (GJ), 20.VI.1992, R.S.; 8 specs., Trăisteni, valley of Doftana River (PH), 2228.VI.1994, A.P.; 1 spec., Cârțișoara, Vama Cucului (SB), 30.VII.1994, C.H.; Bârsana (MM), 5.VII.1995, I.M., C.P. (3 specs), 21.VII.1997, C.P. (8 specs); 17 specs Pietrosu Mt. (National Park "Pietrosu Rodnei", 800-1,300 m a.s.l. (MM), 11-12.VII. 1995, M.I., A.S.; National Park "Pietrosu Rodnei", Laboratory house, 1,370 m, Rodna Mts (MM), 11-13.VII.1995, P.C. (26 specs), 12.1X.1996, C.P., (20 specs); Moisei, Izvorul 
lui Dragoș f.c. (MM), 13.VII. 1995, A.S. (4 specs), 21.VII. 1997, R.E. (4 specs); 1 spec., Strâmtura, Slătioara River, Berșotă stream (MM), 7.1X.1995, C.P.; 1 spec. Ieud (MM), 8.1X.1995, C.P.; 9 specs, Izvorul Izei (MM), 9.IX. 1995, C.P.; 32 specs, Săpânța, Colibi f.c. (MM), 12.VI.1996, I.M.; 1 spec., Valley of Azuga River (PH), 31.VII.1996; 21 specs, Predeal (BV), 3.V.1997, D.M.; 2 specs Smereceni clearing, 7 km upstream Repedea (MM), 22-25.VI.1997, I.M., C.P.; 96 specs, Cârligătura valley, 17 km upstream Repedea (MM), 27.VI.1997, M.I., A.S.; 2 specs, Simionești, Valley of Budaș River (BN), 28.VI.1997, D.R.; 1 spec. Bălăsâna Valley, 4 km upstream Baia Borșa (MM), 21.VIII.1997, E.R.; 1 spec. Valley of Frumușeaua River, Crasna Vișeului (MM), 29.VIII.1997, C.P.; 1 spec., Desești, 2 krn upstream Mara, Colnic f.c. (MM), 17.VII.1998, I.M.; 7 specs, Cornești, left bank of the river Coșeu (MM), 19. VII.1998, V.A.; 1 spec., Izvoarele resort (MM), 23.VII. 1998, I.M.; 2 specs, Piscul cu Brazi Valley, Piatra Craiului Mts, 5.VII.2000, L.O.P.; 11 specs, Piatra Craiului Mts, "Valea Scheilor" chalet (AG), 5.VII.2000, A.S.; 9 specs, Piatra Craiului Mts, Dragoslăveni Valley, 8.VII.2000, C.P., A.V.; Gura Bârsei chalet, Piatra Craiului Mts (BV), 9.VI.2000. C.P. (3 specs), 14.VI.2001, C.P. (3 specs), 2.VII.2002, C.P. (1 spec.); 1 spec., Galeș (AG), 8.VII.2001, C.P.; 5 specs, Bârsa Tămașului, Piatra Craiului Mts (BV), 9.VI.2002, C.P; 3 specs, Șpirla Valley, Piatra Craiului Mts (BV), 3.VII.2002, C.P.; 5 spec. Poienile de sub Munte, the confluence of the rivers Rica and Budescu (MM), 14.VI.2003, C.P.; 3 specs, Poienile de sub Munte, Coșnea f.c. (MM), 15-16.V1.2003, C.P.; 7 specs, Valley of the Pop Ivan River, $11 \mathrm{~km}$ upstream Crasna Vișeului (MM), 17.VI.2003, C.P.; 2 specs, Repedea f.c., Repedea (MM), 18.VI.2003, C.P.; Făina, Valley of the Vaser River (MM), 19.VI.2003, C.P., R.S., M.S. (9 specs), 21.VII.2004, C.B. (1 spec.); 1 spec. Dragomirești, Valley of Baicu River (MM), 21.VI.2003, C.P.; 25 specs, Baicu f.c., Dragomirești, Tibleș Mts, (MM), 21-22.VI.2003, R.S., M.S., D.S.; 1 spec., Valley of the streamlet Pălcuț, Dragomirești, Țibleș Mts (MM), 22.VI.2003, D.S.; 22 specs, Lunca lui Cosiță, $4 \mathrm{~km}$ upstream Baicu f.c., the confluence of the rivers Idișor and Baicu, Dragomirești (MM), 22.VI.2003, R.S.; 1 spec., Săliștea, Valley of the streamlet Idișor, Țibleș Mts (MM), 23.V1.2003, D.S.; 43 specs, National Park Piatra Craiului Mts, Sătic, Onceoaia Valley (AG), 23.V.2004, C.B., R.S.; 6 specs, National Park Piatra Craiului Mts, Bârsa Fierului Valley (BV), 24.V.2004, S.R.; 3 specs, Sărata (SB), 24.VI.2004, C.B.; 2 specs, the confluence of the Pop Ivan and Hututeanca rivers (MM), 18.VII.2004, R.S.; 6 specs, Valley of the Vaser River, Bardău-Cozia transect (MM), 22.VII.2004, C.P.; 3 specs, Valley of the Vaser River, Făina-Bardău transect (MM), 22.VII.2004, C.B.; 1 spec., Predeal, Bucegi Mts (BV), 9.VII.2004, V.U.; 1 spec., Pârâul Rece, Bucegi Mts (BV), 9.VII.2004, V.U.; 1 spec., the confluence of the rivers Vișeu and Tisa (MM), 24.VII.2004, C.P.; 18 specs, the Oticu River Valley, Făgărass Mts, 4.VIII.2004, R.S.; 7 specs, Făgăraș Mts, Valley of Buda River (AG), 4.VIII.2004, R.S., A.P.; 1 spec., Făgăraș Mts, Vidraru Lake, "Valea cu Pești" chalet (AG), 6.VIII.2004, R.S.; 12 specs, Pasărea Lake (IF), 2.V.2006, C.P.; 1 spec., Preluca (MM), 12.VI.2007, C.P.; 1 spec., "Frumoasa" Valley (AB), 6.VIII.2014, C.C.; 21 specs, Sadu Valley (SB), 20.V.2015, C.C., Mi.S., E.I., L.M.; 6 specs, Piatra Mare Mts (BV), 26.VII.2015, Vi.G.; 1 spec., Dobra Valley (AB), 3.VI.2016, C.A.; 8 specs, Omu Peak (Omu Hut) - Cerbului Valley route, Bucegi Mts (PH), 7.VII.2016, Vi.G.; 8 specs, Ciungetu Mt., Călimani National Park (SV), 26.VII.2017, Vi.G.; 4 specs, Rețitiș Mt., Călimani National Park (SV), 27.VII.2017, Vi.G.

Host plants: Polygonaceae (Rumex acetosella, R. obtusifolius, $R$. alpinus, Polygonum aviculare, P. amphibium, P. hydropiper), Scrophulariaceae (Linaria vulgaris). 
Subtribe Phratorina Motschulsky, 1860

Phratora Chevrolat, 1836

Phratora (s. str.) tibialis tibialis (Suffrian, 1851)

Material: 279 specs, Bârsana (MM), 5.VII.1995, C.P., I.M.; 1 spec., Vadul Izei (MM), 9.VII.1995, I.M.; 9 specs, Nănești (MM), 10.VII.1995, I.M.; 2 specs, Văleni (Călinești), "Săcături” f.c. (MM), 7.VII.1995, C.P.; 2 specs, Pietrosu Mt. (National Park "Pietrosu Rodnei", 1500-2050 m), 12.VII.1995, I.M.; 2 specs,. Strâmtura, Slătioara River, Berșotă stream (MM), 7.IX.1995, C.P.; 2 specs, Izvorul Izei (MM), 9.IX.1995, C.P.; 1 spec., Desești, Săpânța, Colibi f.c. (MM), 800 m, 8.VII.1996, I.M.; 3 specs, Celic Dere Monastery (TL), 24.V.1997, C.H., 1 spec., Repedea f.c., Repedea (MM), 29.VI.1997, A.S.; 1 spec., Moisei (MM), 21.VIII.1997, C.P.; 14 specs, Mara, 1 km upstream Mara (MM), 21.VII.1998, I.M.; 1 spec., Dragomirești, Valley of Baicu River (MM), 21.VI.2003, C.P.; 2 specs, Valley of the Socolău River, $2 \mathrm{~km}$ upstream of the confluence of the Socolău and Rica rivers (MM), 19.VII.2004, R.S.; 1 spec., Bârsa Valley, $2 \mathrm{~km}$ downstream Plaiul Foii, The National Park "Piatra Craiului" (BV), 24.V.2004, R.S. (Fig. 5).

Host plants: Salicaceae (Salix sp.) (Mohr, 1966).

Phratora (s. str.) vitellinae (Linnaeus, 1858)

Material: 1 spec., Valley of Frumușeaua River, 7 km upstream Crasna Vișeului (MM), 25.VI.1997, A.S.; 1 spec., Poienile de sub Munte, $500 \mathrm{~m}$ downstream the confluence of the rivers Rica and Budescu (MM), 14.VI.2003, C.P.; 1 spec., Leordina (MM), 15.VI.2003, M.S.; 5 specs, National Park Piatra Craiului Mts, Sătic, Onceoaia Valley (AG), 23.V.2004, R.S.; 1 spec., Valley of the Socolău River, 2 km upstream of the confluence of the Socolău and Rica rivers (MM), 19.VII.2004, R.S.; 1 spec., the confluence of the rivers Vișeu and Tisa (MM), 24.VII.2004, C.P.

Host plants: Salicaceae (Salix sp., Populus sp.) (Mohr, 1966).

Subtribe Prasocurina Reitter, 1913

Neophaedon Jakobson, 1901

Neophaedon segnis Weise, 1884

Material: 1 spec., Rodna Mts, Corongiș Peak (MM), without other data, coll. Fr.D. (Fig. 6). 2010).

Host plants: Caryophyllaceae (Malachium sp., Stellaria sp.) (Warchałowski,

Phaedon Latreille, 1829

Phaedon (s. str.) armoraciae (Linnaeus, 1758)

Material: 2 specs, Brașov Mts., without other data, coll. Fr.D.; 1 spec., Dunavățu (TL), 9.VI.1994, C.H.; 1 spec., Vadu Izei (MM), 9.VII.1995, I.M.; 1 spec., Poienile de sub Munte, Valley of Budescu River (MM), 14.VI.2003, M.S.

Host plants: Scrophulariaceae (Veronica anaglallis) (Warchałowski, 2010).

Phaedon (s. str.) cochleariae cochleariae (Fabricius, 1792)

Material: 2 specs, Smereceni clearing, 7 km upstream Repedea (MM), 22-27. VI.1997, R.S. A.S.; 1 spec., Repedea f.c., Repedea (MM), 26.VI.1997, I.M.; 1 spec., Canaraua Fetii (CT), 10.IV.1999, M.S.P.; 1 spec., the confluence of Vișeu and Tisa 


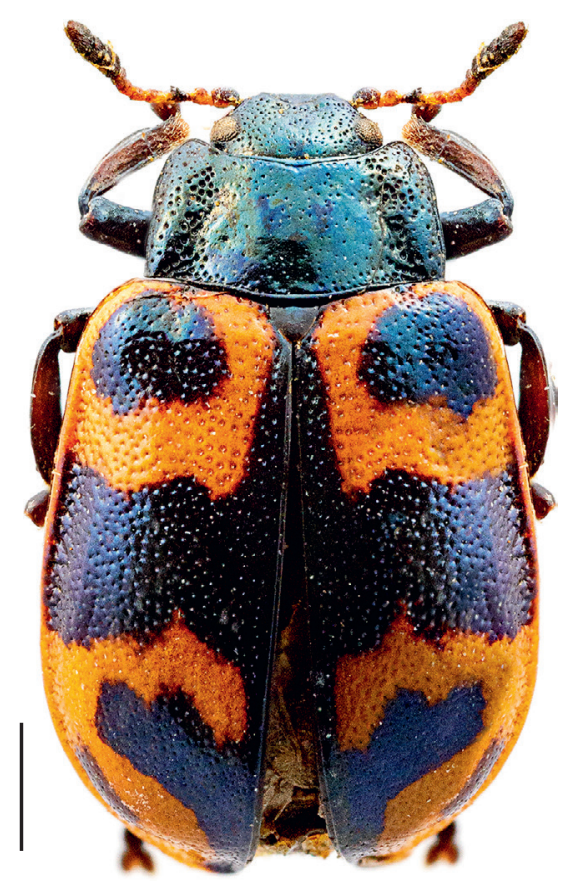

Fig. 4 - Chrysomela lapponica. Scale bar $1 \mathrm{~mm}$.

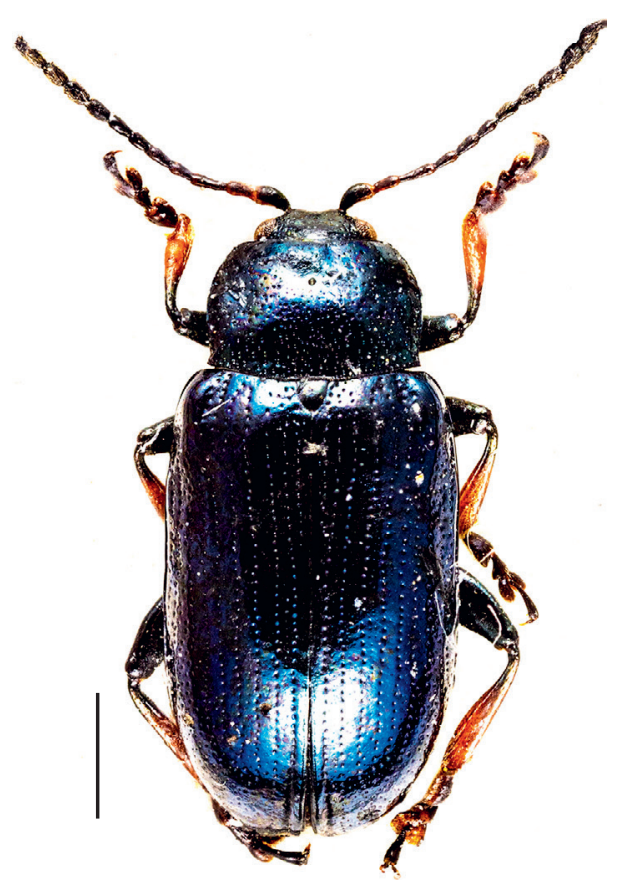

Fig. 5 - Phratora tibialis. Scale bar $1 \mathrm{~mm}$. 
rivers (MM), 24.VII.2004, C.P.; 1 spec., Bordușani (IL), 21.VIII.2006, E.I.; 1 spec., France, Pommera, without other data, coll. Ed.R. (Fig. 7).

Host plants: Brassicaceae (Nasturtium officinale, Roripa amphibia) (Mohr, 1966).

Phaedon (s. str.) laevigatus laevigatus (Duftschmid, 1825)

Material: 1 spec., Valea Oltului (Altfluss), without other data, coll. Fr.D.

Host plants: Lamiaceae (Galeopsis pubescens, G. ladanum) (Mohr, 1966).

Prasocuris Latreille, 1802

Prasocuris (Hydrothassa) glabra (Herbst, 1783)

Material: 3 specs, Postăvaru Mts (Schuler Gebirge) (BV), without other data, coll. Fr.D.; 1 spec., Baicu f.c., Ţibles Mts, Dragomirești (MM), 22.VI.2003, C.P. (Fig. 8).

Host plants: Ranunculaceae (Ranunculus sp., Caltha sp.) (Warchałowski, 2010).

Prasocuris (Hydrothassa) marginella marginella (Linnaeus, 1758)

Material: 2 specs., Postăvaru Mts (Schuler Gebirge) (BV), without other data, coll. Fr.D.; 1 spec., Deia, Câmpulung Moldovenesc (SV), 3.XI.1951, coll. I.C.; 1 spec., Broșteni (SV), 27.V.1958; 1 spec., Făina, Valley of Vaser River (MM), 21.VII.2004, C.P. (Fig. 9).

Host plants: Ranunculaceae (Ranunculus sp., Caltha sp.) (Warchałowski, 2010).

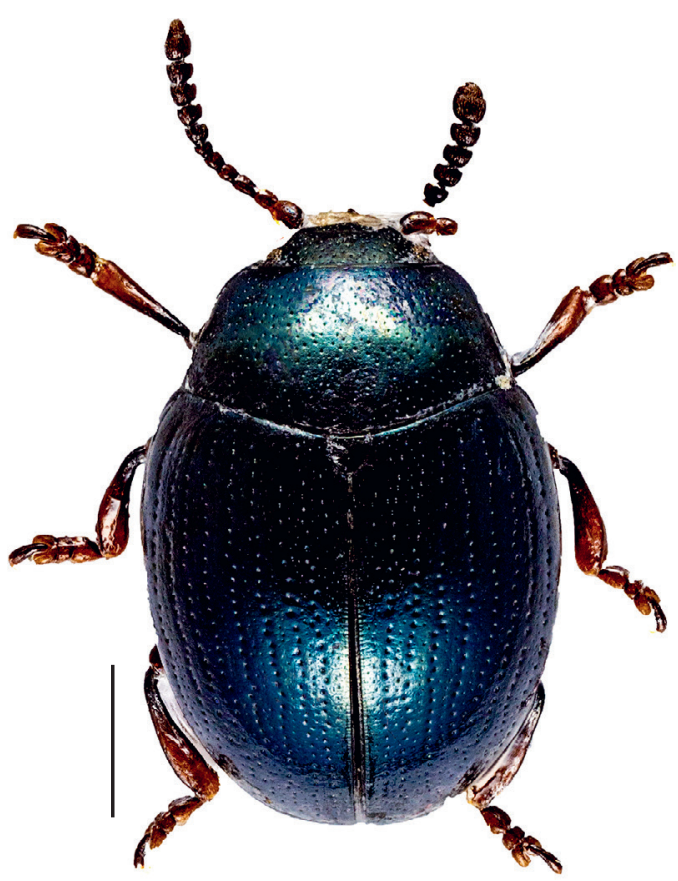

Fig. 6 - Phaedon segnis. Scale bar $1 \mathrm{~mm}$.

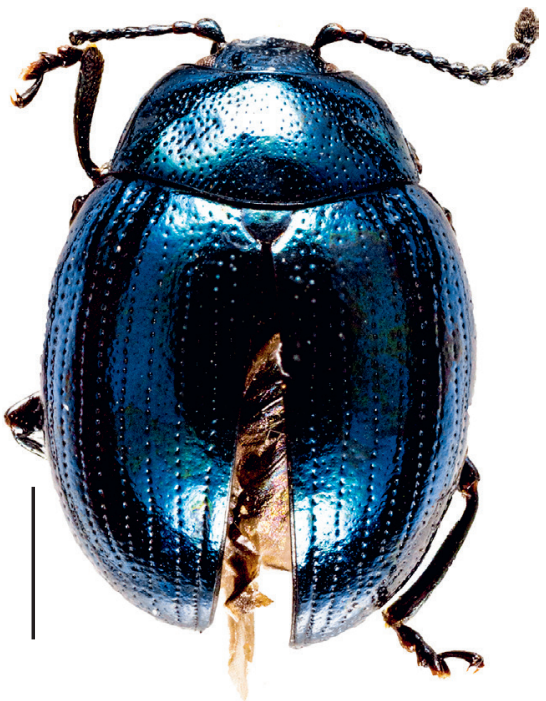

Fig. 7 - Phaedon cochleariae. Scale bar $1 \mathrm{~mm}$. 


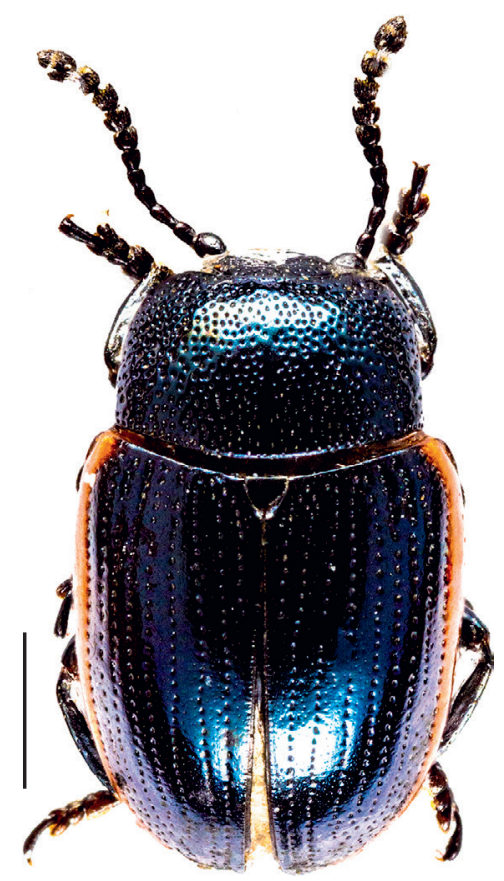

Fig. 8 - Prasocuris glabra. Scale bar $1 \mathrm{~mm}$.

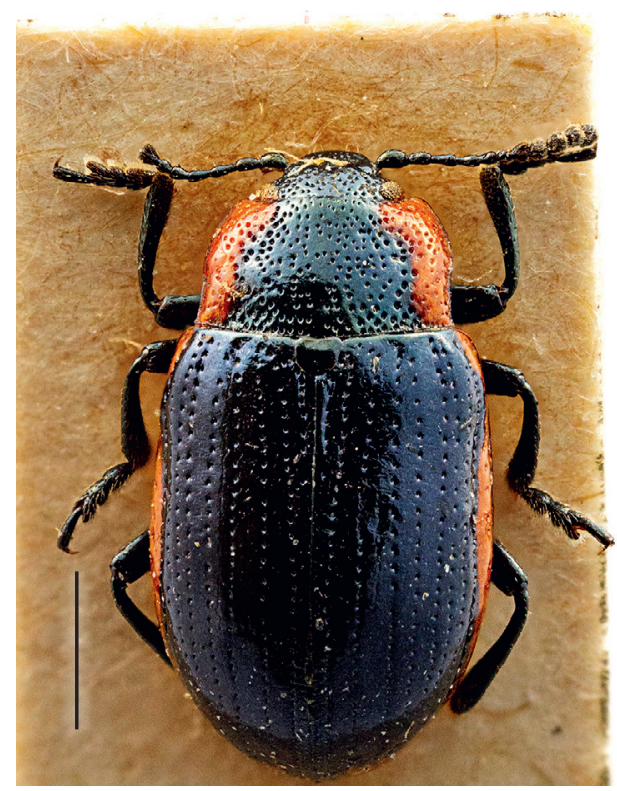

Fig. 9 - Prasocuris marginella. Scale bar $1 \mathrm{~mm}$.

Prasocuris (s. str.) junci (Brahm, 1790)

Material: 2 spec., Șpring (AB), without other data, coll. I.C. (Fig. 10).

Host plants: Primulaceae (Anagallis sp.), Scrophulariaceae (Veronica beccabunga) (Mohr, 1966).

\section{Prasocuris (s. str.) phellandrii Linnaeus, 1758}

Material: 1 spec., Titu (DB), VIII., coll. R.C.; 1 spec., Periprava, Danube Delta (TL), 9.V.1964, M.W.; 1 spec., Eșelnița (MH), 3.IV.1968, S.; 1 spec., Foieni (SM), 24.IV.1986, C.P.; 1 spec., Poiana Neamțului (SB), 15.V.1986, I.D.; 1 spec., Taița Lake, Horia (TL), 26.V.2005, I.M. (Fig. 11).

Host plants: Apiaceae (Oenanthe sp., Sium sp., Cicuta sp., Berula sp.) (Warchałowski, 2010).

In the "Grigore Antipa" National Museum's collections 21 species of the Chrysomelini tribe belonging to eight genera are preserved: Chrysomela (seven species), Plagiodera, Plagiosterna, Neophaedon (each with one species), Gastrophysa and Phratora (each with two species), Phaedon (three species) and Prasocuris (four species), representing over $90 \%$ of the total number of species recorded in the Romanian fauna.

All species of the Chrysomela, Plagiodera, Plagiosterna, Gastrophysa and Prasocuris genera reported in Romania are preserved in the museum's collection (Maican, 2005; Kippenberg, 2010).

Among the rare species in Romanian fauna, stored in this collection, Chrysomela lapponica, Prasocuris junci and Chrysomela cuprea should be mentioned. 


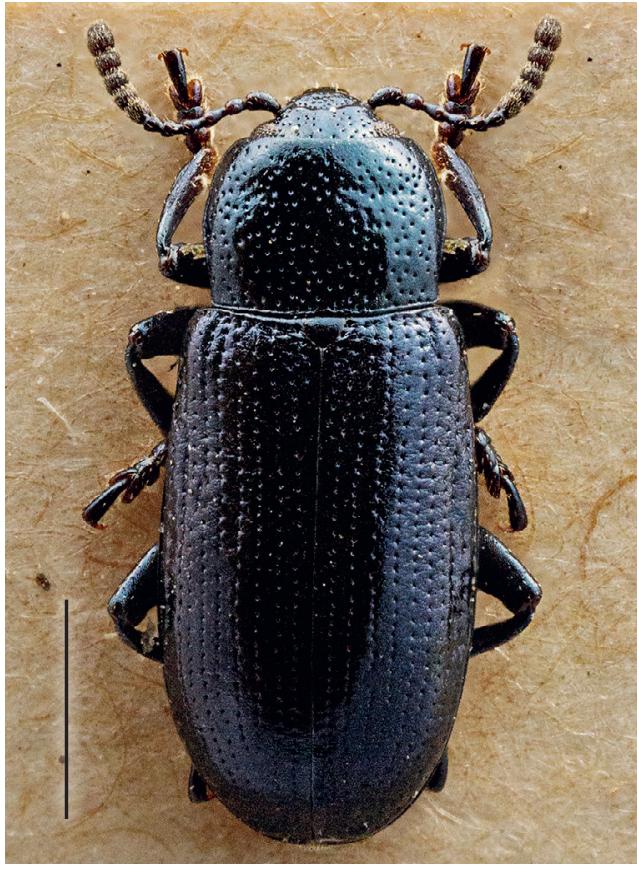

Fig. 10 - Prasocuris junci. Scale bar $1 \mathrm{~mm}$.

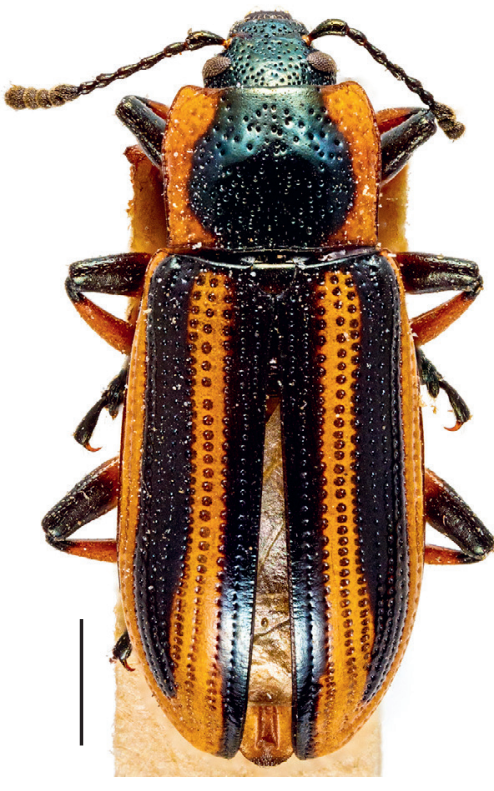

Fig. 11 - Prasocuris phellandrii. Scale bar $1 \mathrm{~mm}$.

Most part of the material originates in Romania, but the collection also includes specimens from Germany (belonging to species Plagiodera versicolora), France (Phaedon cochleariae, Chrysomela populi), Serbia (Chrysomela vigintipunctata vigintipuncatata), Turkey and Syria (Gastrophysa polygoni).

Also, the material of Chrysomelini preserved in the old Collection of Palaearctic Coleoptera of "Grigore Antipa" Museum has been reviewed. This collection includes specimens donated or aquired by the museum specialists from different areas of the Palaearctic region. It was created by gathering all the collections entered into the scientific heritage of the museum, at the end of the $19^{\text {th }}$ century and the beginning of the $20^{\text {th }}$ century. The Collection of Palaearctic Coleoptera stores specimens belonging to six species which are not found in the recently established Chrysomelidae Collection, namely: Phratora vulgatissima (Linnaeus, 1758), P. atrovirens (Cornelius, 1857), $P$. laticollis (Suffrian, 1851), Neophaedon pyritosus (Rossi, 1792), Phaedon tumidulus (Germar, 1824) and Prasocuris hannoveriana (Fabricius, 1775).

\section{ACKNOWLEDGMENTS}

The authors express thanks to Dr. Melanya Stan, deputy director of "Grigore Antipa" National Museum of Natural History, for granting access to the collections, and to Dr. Jan Bezděk (Mendel University, Brno) for his constructive comments on the manuscript. Many thanks to Dr. Ionuţ Ştefan Iorgu and Dr. Tiberiu Sahlean for photos, and also to Rada Tiţă, Marinela Năzăreanu, Marcela Bugnar, Mariana Foaltin and Dana Ciopec for their help in preparation and labeling of the specimens, and the proper conservation of collections. A part of this study was funded by project no. RO1567-IBB01/2018 from the Institute of Biology Bucharest of Romanian Academy. 


\section{REFERENCES}

BOUCHARD, P., Y. BOUSQUET, A. E. DAVIES, M. A. ALONSO-ZARAZAGA, J. F. LAWRENCE, C. H. C. LYAL, A. F. NEWTON, C. A. M. REID, M. SCHMITT, S. A. ŚLIPIŃSKI, A. B. T. SMITH (2011) Family-group names in Coleoptera (Insecta). Zookeys, 88: 1-972.

HOINIC, C. (1994) A review of the species of Macroplea Samouelle (Coleoptera: Chrysomelidae) in Romania. Travaux du Muséum d'Histoire Naturelle "Grigore Antipa", 34: 17-30.

KIPPENBERG, H. (2010) Subfamily Chrysomelinae Latreille, 1802. Pp. 390-442. In: I. Löbl, A. Smetana (eds) Catalogue of Palaearctic Coleoptera, Chrysomeloidea, 6, Apollo Books, Stenstrup, 924 pp.

MAICAN, S. (2005) Checklist of Chrysomelidae (Coleoptera) of Romania. Travaux du Muséum National d'Histoire Naturelle "Grigore Antipa", 48: 119-136.

MAICAN, S. (2006) Clytrins (Coleoptera: Chrysomelidae: Clytrinae) from the collections of the "Grigore Antipa" National Museum of Natural History (Bucharest). Travaux du Muséum National d'Histoire Naturelle "Grigore Antipa", 49: 239-257.

MAICAN, S. (2007a) Some Mediterranean Chrysomelids species (Coleoptera: Chrysomelidae) newly entered in the collections of "Grigore Antipa" National Museum of Natural History [Results of expeditions from Turkey and Tunisia, 2005-2006]. Travaux du Muséum National d'Histoire Naturelle "Grigore Antipa", 50: 421-429.

MAICAN, S. (2007b) Contributions to the knowledge of the leaf beetle fauna (Coleoptera: Chrysomelidae) from Maramureş (northern Romania). Entomologica Romanica, 12: 301-324.

MAICAN, S., R. SERAFIM (2001) Chrysomelidae (Coleoptera) from Maramureş. Travaux du Muséum National d'Histoire Naturelle "Grigore Antipa", 43: 199-233.

MAICAN, S., R. SERAFIM (2004) Leaf-beetles (Coleoptera: Chrysomelidae) from Maramureş (Romania). Travaux du Muséum National d'Histoire Naturelle "Grigore Antipa", 46: 139-159.

MAICAN, S., R. SERAFIM (2009) Data about Cerambycidae and Chrysomelidae (Coleoptera: Chrysomeloidea) collected from Mediterranean expeditions [Results of the "Taurus" 2005, "Focida" 2006, "Punia" 2006, "Atlas" 2007 and "Euphrates" 2008 expeditions]. Travaux du Muséum National d'Histoire Naturelle "Grigore Antipa", 52: 415-428.

MAICAN, S., R. SERAFIM (2012) Overview on the Chrysomeloidea superfamily (Coleoptera: Cerambycidae, Orsodacnidae, Chrysomelidae) in Dobrogea (Romania). Travaux du Muséum National d'Histoire Naturelle "Grigore Antipa", 55 (1): 65-123.

MAICAN S., R. SERAFIM (2016) The catalogue of Donaciinae and Criocerinae species (Coleoptera: Chrysomelidae) from the new leaf beetles collection from "Grigore Antipa" National Museum of Natural History (Bucharest) (Part I). Travaux du Museum National d'Histoire Naturelle "Grigore Antipa", Bucharest, 59 (2): 179-194.

MAICAN S., R. SERAFIM (2017) Catalogue of Cassidinae (Coleoptera: Chrysomelidae) from the New Leaf Beetles Collection from "Grigore Antipa" National Museum of Natural History (Bucharest) (Part II). Travaux du Museum National d'Histoire Naturelle "Grigore Antipa", Bucharest, 60 (2): 477-494.

MOHR, K.-H. (1966) Familie Chrysomelidae. Pp. 95-280. In: Freude, H., K. W. Harde, G. A. Lohse (eds.), Die Käfer Mitteleuropas, Band 9, Cerambycidae, Chrysomelidae, Goecke \& Evers Verlag, Krefeld, 299 pp.

NEGRU, ŞT. (1968) Ord. Coleoptera (pars). In: Entomofaune de l'ile de Letea (Delta du Danube). Travaux du Muséum d'Histoire Naturelle "Grigore Antipa", 9: 81-95. (In French)

NEGRU, ŞT., A. ROŞCA (1967) Ord. Coleoptera. In: L'entomofaune des forêsts du sud de la Dobroudja. Travaux du Muséum d'Histoire Naturelle "Grigore Antipa”, 7: 119-148. (In French)

REID, C.A.M (2014). Chrysomelinae Latreille, 1802. Pp. 243-251. In: Leschen R.A.B., R.G. Beutel (eds.) Coleoptera Beetles, Vol. 3, Morphology and Systematics (Phytophaga). Walter de Gruyter GmbH \& Co KG, Göttingen, 675 pp.

SERAFIM, R., S. MAICAN (2004) Contributions to the knowledge of the Coleopterans from the littoral of the Black Sea (Romania). Travaux du Muséum National d'Histoire Naturelle "Grigore Antipa", 47: 169-210.

SERAFIM, R., S. MAICAN (2008) Data on Cerambycidae and Chrysomelidae (Coleoptera: Chrysomeloidea) from Bucureşti and surroundings. Travaux du Muséum National d'Histoire Naturelle "Grigore Antipa", 51: 387-416.

SERAFIM, R., S. MAICAN (2011) Catalogue of Cerambycidae, Megalopodidae and Chrysomelidae (Coleoptera: Chrysomeloidea) recently entered in the patrimony of "Grigore Antipa" National Museum of Natural History (Bucharest). Igor Ceianu collection. Travaux du Muséum National d'Histoire Naturelle "Grigore Antipa", 54 (2): 425-460. 
STAN, M., R. SERAFIM, S. MAICAN (2016) Data on the Beetle Fauna (Insecta: Coleoptera) in "Frumoasa" Site of Community Importance (ROSCI0085, Romania) and Its Surroundings. Travaux du Muséum National d'Histoire Naturelle "Grigore Antipa", 59 (2): 129-159.

UNGUREANU, V., S. MAICAN, R. SERAFIM (2008) Diversity of coleopterans: Cerambycidae, Chrysomelidae, Coccinellidae from Buzău area (Romania). Travaux du Muséum National d'Histoire Naturelle "Grigore Antipa", 51: 171-183.

WARCHAŁOWSKI, A. (2003) Chrysomelidae. The leaf beetles of Europe and the Mediterranean area. Natura Optima dux. Foundation, Warszawa, $600 \mathrm{pp}$.

WARCHAŁOWSKI, A. (2010) The Palaearctic Chrysomelidae. Identification keys. $1 \& 2$. Warszawa, $1212 \mathrm{pp}$. 